\title{
Gait Analysis of Old Individuals with Mild Parkinsonian Signs and Those Individuals' Gait Performance Benefits Little from Levodopa
}

This article was published in the following Dove Press journal: Risk Management and Healthcare Policy

Zhuang Wu $\mathbb{D}^{\prime}, *$
Hang Xu',
Sha Zhu'
Ruxin Gu'
Min Zhong'
Xu Jiang'
Bo Shen'
Jun Zhu'
Yang Pan'
Jingde Dong $\mathbb{D}^{\prime}$
Jun Yan'
Wenbin Zhang $\mathbb{D}^{3}$
Li Zhang'

'Department of Geriatric Neurology, Affiliated Brain Hospital of Nanjing Medical University, Nanjing, People's Republic of China; ${ }^{2}$ Department of Neurology, The Yancheng Clinical College of Xuzhou Medical University, Yancheng, People's Republic of China; ${ }^{3}$ Department of Neurosurgery, Affiliated Brain Hospital of Nanjing Medical University, Nanjing, People's Republic of China

*These authors contributed equally to this work
Background and Purpose: Gait analysis and the effects of levodopa on the gait characteristics in Mild parkinsonian signs (MPS) are rarely published. The present research aimed to (1) analyze the gait characteristics in MPS; (2) explore the effects of levodopa on the gait performance of MPS.

Methods: We enrolled 22 inpatients with MPS and 20 healthy control subjects (HC) from Nanjing Brain Hospital. The Unified Parkinson's Disease Rating Scale was used to evaluate motor symptoms. Acute levodopa challenge test was performed to explore the effects of levodopa on the gait performance of MPS. The instrumented stand and walk test was conducted for each participant and the JiBuEn gait analysis system was used to collect gait data.

Results: For spatiotemporal parameters: Compared with HC, the state before taking levodopa/benserazide in MPS group (meds-off) demonstrated a decrease in stride length (SL) $(p \leq 0.001)$, an increase in SL variability $(p \leq 0.001)$, and swing phase time variability $(p=0.016)$. Compared with meds-off, the state after 1 hour of taking levodopa/benserazide in MPS group (meds-on) exhibited an increase in SL $(p \leq 0.001)$, a decrease in SL variability $(p \leq 0.001)$. For kinematic parameters: Compared with HC, meds-off demonstrated a decrease in heel strike angle $(p=0.008)$, range of motion ( $\mathrm{ROM})$ of knee joint $(p=0.011)$ and ROM of hip joint $(p=0.007)$. Compared with meds-off, meds-on exhibited an increase in HS $(p \leq 0.001)$. Bradykinesia and rigidity scores were significantly correlated with gait parameters.

Conclusion: Although the clinical symptoms of the MPS group are mild, their gait damage is obvious and they exhibited a decreased SL and joints movement, and a more variable gait pattern. Levodopa had little effect on the gait performance of those individuals.

Keywords: elderly, mild parkinsonian signs, gait, levodopa challenge test

\section{Introduction}

The typical clinical manifestations of patients with Parkinson's Disease (PD) include bradykinesia, rest tremor and rigidity. However, some elderly individuals who do not meet the established diagnostic criteria for PD or any other neurodegenerative diseases exhibit one or some combination of the above clinical symptoms. These symptoms are described as mild parkinsonian signs (MPS) and the incidence of MPS among old individuals ranges from $15 \%$ to $95 \%{ }^{1-3}$ The life quality of people with MPS often decreases. ${ }^{4}$ Old individuals with MPS often present a low-level ability in performing physical activities. ${ }^{5}$ MPS is often associated with cognitive decline, ${ }^{6}$
Correspondence: Li Zhang; Jun Yan Email neuro_zhangli@163.com; 2519002414@qq.com
Risk Management and Healthcare Policy 2021:|4 II09-I I I8 
dementia, $^{2}$ depression ${ }^{7}$ and risk of falling. ${ }^{8}$ Moreover, old individuals with MPS has a higher mortality and higher institutionalization rate than normal ones. ${ }^{1,9}$ Therefore, the proper management of MPS is a priority for clinicians. Substantial research has focused on the gait performance of patients with PD in the past, but only a few has conducted gait analysis on people with MPS. ${ }^{10}$ Previous studies confirmed that the PD fallers peak velocity of the trunk decreased significantly which can be employed to discriminate faller from non-faller PD patients. ${ }^{11}$ The loss of substantia nigra neurons result in PD. The putamen dopaminergic innervation in PD patients was significantly correlated with the center of pressure, first step velocity and length, and the center of mass velocity and acceleration. Levodopa can improve above gait parameters too. ${ }^{12}$ However, the etiopathogenesis that underlies MPS remains unknown. Pigmented neurons of nigra have been reported to decline at a speed of $4.7 \%$ per decade in early adulthood. ${ }^{13}$ To what extent this age-associated dopamine loss accounts for the development of MPS is still unknown. ${ }^{1,14}$ The following questions need to be addressed urgently: What effects does levodopa have on the gait characteristics in MPS? Can old individuals with MPS benefit from levodopa? ${ }^{15}$ The acute levodopa challenge test (ALCT) can be used to predict long-term levodopa responsiveness. ${ }^{16}$ Accordingly, to understand the gait performance and the pathogenesis of MPS, we conducted an experiment to: (1) analyze the gait characteristics of MPS comprehensively; and (2) explore the effects of levodopa on the gait performance of old individuals with MPS. We hypothesized that MPS group may exhibit an impaired gait pattern and those individuals' gait performance can be improved to some extent after taking levodopa. Our research will aid the management of elderly individuals with MPS, improve the quality of their life, and shed more light on the pathogenesis of MPS.

\section{Methods}

\section{Participants}

In our research, we enrolled 22 inpatients with MPS and 20 healthy control subjects (HC) from the Department of Geriatrics, Affiliated Brain Hospital of Nanjing Medical University between October 2019 and August 2020. The severity of symptoms in patients with MPS was assessed from the following three domains in part III (the motor part) of the Unified Parkinson Disease Rating Scale (UPDRS): (1) Bradykinesia in extremities was evaluated using UPDRS subitems \#23-26 and subitem \#31; (2) Rest tremor was assessed using UPDRS subitem \#20; (3) Rigidity in neck and extremities was evaluated using UPDRS subitem \#22. Inclusion criteria for MPS were as follows: (1) a score of any one of the three domains (bradykinesia, rigidity, or tremor) greater than zero; (2) no medical history of PD; (3) ability to follow doctors' instructions; (4) a Mini-Mental State Examination (MMSE) score $>24$. Exclusion criteria for MPS were as follows: (1) meeting the diagnostic criteria of PD, hepatolenticular degeneration, Alzheimer's disease, dementia with Lewy bodies, and multiple system atrophy; (2) other conditions that could influence gait performance, including cerebrovascular disease, and spinal column diseases. Inclusion criteria for $\mathrm{HC}$ were as follows: (1) no medical history of $\mathrm{PD}$, hepatolenticular degeneration, Alzheimer's disease, dementia with Lewy bodies, and multiple system atrophy, cerebrovascular disease, spinal column diseases that may influence gait performance; (2) an MMSE score $>24$. The Medical Ethics Committee of the Affiliated Brain Hospital of Nanjing Medical University approved the experimental procedures. All participants signed written informed consent and all experimental procedures were in accordance with the declaration of Helsinki.

\section{Clinical Evaluation}

The following baseline data were collected for all participants: gender, age, height, weight, body mass index (BMI), shoe size and MMSE score. For all participants, the UPDRS part III was used to evaluate motor symptoms.

\section{Acute Levodopa Challenge Test}

ALCT was performed in the MPS group to investigate their response to levodopa. All the following procedures were performed in the morning and all participants were instructed to fast before ALCT. In this test, each participant of the MPS group was instructed to take $250 \mathrm{mg}$ of Madopar (200mg levodopa and 50mg benserazide, Roche Pharma AG, Basel, Switzerland). The state before taking levodopa/benserazide was defined as the OFF state (medsoff). At this time, two experienced neurology clinicians conducted the first UPDRS assessment separately, and the results of evaluation were averaged. The state after 1 hour of taking levodopa/benserazide was defined as the ON state (meds-on). At this time, the second UPDRS assessment was conducted, and the results were also averaged. For ethical reasons, ALCT was not performed in the HC 
group. All subjects in the HC group only underwent one UPDRS assessment on an empty stomach.

\section{Gait Data Collection}

In this study, the JiBuEn gait analysis system was applied in gait data collecting. This set of gait analysis system was consisted of inertial micro-electro-mechanical system sensors which were bound to patient's lower limbs (the lower and upper sides of the knee joint) and waist and a pair of smart shoes. The system collected motion signals and transmitted gait data to computer and the final gait data were obtained. The zero-correction algorithm, hexahedral calibration technique, and high-order low-pass filter were used in data preprocessing which can decrease high-frequency noise and reduce accumulative errors. The accuracy of this equipment has been verified before. ${ }^{17}$ The following spatiotemporal gait parameters were collected: stride length (SL), cadence (CA), gait velocity (GV), stride time (ST), swing phase time (SwPT), and stance phase time (StPT). In addition, we measured SL variability (CV-SL), ST variability (CV-ST), SwPT variability (CV-SwPT), StPT variability (CV-StPT). The following Kinematic gait parameters were collected: the range of motion (ROM) of ankle joints (AJ), knee joints (KJ), and hip joints (HJ). We also included toe-off (TO) and heel strike (HS) angle in data analysis. All participants performed the instrumented stand and walk test (ISAW). The detailed procedures of ISAW were as follows: ${ }^{18}$ First, all participants were instructed to stand for 30 seconds with arms at their side, then walked in a comfortable way for $7 \mathrm{~m}$, turned $180^{\circ}$, and walked back to the initial place. At the same time, we used JiBuEn gait to collect gait data.

\section{Statistical Analysis}

Quantitative data were shown as mean \pm Standard Deviation (SD). The normality of distribution of clinical characteristics and gait data were initially tested using the Kolmogorov-Smirnov test.

The corresponding difference of gait data among HC, meds-off, and meds-on were analyzed using one-way analysis of variance initially, when three group gait data followed normal distribution. Otherwise, KruskalWallis $H$-test was used. Then, for normally distributed data, the independent $t$-test or paired $t$ test were performed to compare the differences between two groups. For non-normally distributed gait data of intergroup gait characteristics, the Mann-Whitney $U$-Test or Wilcoxon signed-rank test were used. Spearman correlation analysis was conducted to investigate associations between gait parameters and UPDRS assessment scores. For all analyses, a $P$ value $<0.05$ indicated significant difference. As we used multiple tests, $P$ value of intergroup comparisons among $\mathrm{HC}$, meds-off and meds-on were corrected using the Bonferroni method. The alpha value was set at $P^{\prime}=0.05 /$ times of comparison, that is $P^{\prime}=0.017$. The calculation of gait variability was divided into two steps. First, gait variability from the left and the right were computed separately using Formula (1). Then, the variability on both sides was integrated using Formula (2). ${ }^{19}$ The asymmetry index (AI) was used to assess the symmetry of gait parameters (Formula (3)). ${ }^{20-22}$

Formula (1): $\mathrm{CV}_{\text {separate }}=\mathrm{SD} \div$ mean value

Formula (2): $\mathrm{CV}_{\text {combined }}=\sqrt{\frac{\mathrm{CV}_{\mathrm{L}}+\mathrm{CV}_{\mathrm{R}}}{2}} * 100$

The subscripts $L$ and $R$ indicate the left and right sides of participants, respectively. CV means coefficient of variation.

Formula (3): $\mathrm{AI}=\frac{\max \left(\mathrm{X}_{\mathrm{L}}, \mathrm{X}_{\mathrm{R}}\right)-\min \left(\mathrm{X}_{\mathrm{L}}, \mathrm{X}_{\mathrm{R}}\right)}{\max \left(\mathrm{X}_{\mathrm{L}}, \mathrm{X}_{\mathrm{R}}\right)} * 100$

$X=$ [ST, SL, SwPT, StPT, TO, HS, ROM-HJ, ROMKJ, ROM-AJ], the subscripts $L$ and $R$ represent the left and right sides of participants, respectively. AI: asymmetry index; ST: stride time; SL: stride length; SwPT: swing phase time; StPT: stance phase time; TO: toe-off angle; HS: heel strike angle; ROM: range of motion; HJ: hip joint; KJ: knee joint; and AJ: ankle joint.

IBM SPSS version 25 and GraphPad Prism version 8.0.1 were used in data analysis and figures configuration, respectively.

\section{Results}

\section{Clinical Characteristics of Participants}

In our research, we enrolled 22 inpatients with MPS and 20 healthy controls (HC). Their baseline data are shown in Table 1. No difference was found in all baseline data between two groups. For the MPS group, the mean disease duration was $1.75 \pm 1.38$ years, and the UPDRS III total score in OFF state was 23.55 \pm 14.02 . After ALCT, the UPDRS III improvement rate was $8.23 \% \pm 6.27$. In addition, the score of bradykinesia, rigidity, and rest tremor in the OFF state were $9.91 \pm 7.60,3.77 \pm 4.13$, and $1.55 \pm 1.85$, respectively. 
Table I Clinical Characteristics of Participants

\begin{tabular}{|c|c|c|c|}
\hline & HC & MPS & P-value \\
\hline $\mathrm{N}$ & 20 & 22 & \\
\hline Male(\%) & $9(45)$ & $12(54.55)$ & 0.537 \\
\hline Age(years) & $68.05 \pm 4.55$ & $69.45 \pm 5.21$ & 0.271 \\
\hline Height $(\mathrm{cm})$ & $161.85 \pm 5.40$ & $\mid 65.09 \pm 7.61$ & 0.117 \\
\hline Weight(kg) & $63.10 \pm 5.05$ & $66.18 \pm 8.46$ & 0.165 \\
\hline BMI & $23.46 \pm 2.36$ & $24.29 \pm 2.89$ & 0.315 \\
\hline Shoe size & $39.15 \pm 1.87$ & $40.05 \pm 2.24$ & 0.151 \\
\hline MMSES & $27.20 \pm 2.02$ & $26.73 \pm 1.93$ & 0.406 \\
\hline Disease duration(years) & NA & $1.75 \pm 1.38$ & \\
\hline UPDRS III total score in OFF state & NA & $23.55 \pm 14.02$ & \\
\hline UPDRS III improvement rate(\%) & NA & $8.23 \pm 6.27$ & \\
\hline Three domains total score in OFF state & NA & $15.23 \pm 10.87$ & \\
\hline Domain I-Bradykinesia & NA & $9.91 \pm 7.60$ & \\
\hline Domain 2-Rigidity & NA & $3.77 \pm 4.13$ & \\
\hline Domain 3-Rest tremor & NA & $1.55 \pm 1.85$ & \\
\hline
\end{tabular}

Abbreviations: NA, not applicable; BMI, body mass index; MMSE, mini-mental state examination score; UPDRS III, Unified Parkinson Disease Rating Scale part 3.

\section{Spatiotemporal Gait Parameters}

We found a significant difference in SL ( $p=0.003), \mathrm{CV}-\mathrm{SL}$ $(p \leq 0.001)$, CV-StPT $(p=0.048), \quad$ CV-SwPT $(p=0.040)$ (Table 2). However, for the post hoc analysis of CVStPT, no significant difference was found in intergroup comparisons. Compared with $\mathrm{HC}$, post hoc analysis found a decrease in SL $(p \leq 0.001)$, an increase in CV-SL $(p \leq 0.001)$ and CV-SwPT $(p=0.016)$ in meds-off. Compared with meds-off to meds-on, post hoc analysis demonstrated an increase in SL $(p \leq 0.001)$, and a decrease in CV-SL $(p \leq 0.001)$. Compared with HC, post hoc analysis found a decrease in SL $(p=0.007)$ in meds-on. The walking efficiency analysis of SL and ST are shown in Figure 1 which showed that the distribution of SL and ST in the meds-off group was more dispersed than that of the HC group. In addition, the distribution of SL and ST in the meds-off group tends to be distributed to the upper left in Figure 1.

\section{Kinematic Gait Parameters}

We found statistical difference in HS $(p=0.043)$, ROM-KJ $(p=0.002)$, and ROM-HJ ( $p=0.008)$ (Figure 2). Comparing
$\mathrm{HC}$ to meds-off, post hoc analysis demonstrated a decrease in HS $(p=0.008)$, ROM-KJ $(p=0.011)$ and ROM-HJ $(p=0.007)$. Comparing meds-off to meds-on, post hoc analysis only exhibited an increase in HS $(p \leq 0.001)$. Compared with $\mathrm{HC}$, post hoc analysis found a decrease in ROM-KJ $(p \leq 0.001)$ and ROM-HJ ( $p=0.004)$ in meds-on.

\section{Changes in Gait Parameters After Levodopa/Benserazide Intake in MPS Group}

We observed significant differences in SL, CV-SL, and HS between meds-off and meds-on. Then, we calculated the rate of changes for each of above three gait parameters (Figure 3). Compared with the meds-off state, the SL and HS in meds-on state increased by $\sim 6.19 \%$ and $\sim 11.36 \%$, respectively. The CV-SL for meds-off was $26.88 \pm 5.58$ which was reduced by $\sim 12.17 \%$ in the meds-on state.

\section{Symmetry Analysis of Gait Parameters}

Spatiotemporal and kinematic gait parameters were included in symmetry analysis (Table 3). No difference was found in any of those parameters. 
Table 2 Differences in Spatiotemporal Gait Parameters

\begin{tabular}{|c|c|c|c|c|c|}
\hline & HC & Meds-Off & Meds-On & $P$ & Post Hoc Tests \\
\hline $\mathrm{SL}(\mathrm{m})$ & $1.16 \pm 0.08$ & $0.97 \pm 0.21$ & $1.03 \pm 0.19$ & 0.003 & $\leq 0.00 \mathrm{I}^{\mathrm{a}}, 0.007^{\mathrm{b}}, \leq 0.00 \mathrm{I}^{\mathrm{c}}$ \\
\hline $\mathrm{GV}(\mathrm{m} / \mathrm{s})$ & $0.89 \pm 0.14$ & $0.76 \pm 0.26$ & $0.82 \pm 0.22$ & 0.262 & NA \\
\hline CA(steps/min) & $92.80 \pm 10.91$ & $91.06 \pm 19.98$ & $93.84 \pm 15.98$ & 0.722 & NA \\
\hline ST(s) & $1.31 \pm 0.16$ & $1.35 \pm 0.31$ & $1.34 \pm 0.43$ & 0.723 & NA \\
\hline StPT(s) & $0.85 \pm 0.14$ & $0.91 \pm 0.28$ & $0.90 \pm 0.39$ & 0.800 & NA \\
\hline SwPT(s) & $0.45 \pm 0.06$ & $0.45 \pm 0.04$ & $0.45 \pm 0.04$ & 0.397 & NA \\
\hline CV-SL & $21.27 \pm 2.58$ & $26.88 \pm 5.58$ & $23.61 \pm 4.56$ & $\leq 0.001$ & $\leq 0.00 \mathrm{I}^{\mathrm{a}}, \leq 0.00 \mathrm{I}^{\mathrm{c}}$ \\
\hline CV-ST & $21.81 \pm 5.30$ & $24.64 \pm 8.67$ & $22.10 \pm 7.89$ & 0.233 & NA \\
\hline CV-StPT & $14.78 \pm 2.89$ & $19.11 \pm 8.86$ & $16.36 \pm 4.27$ & 0.048 & ns \\
\hline CV-SwPT & $19.52 \pm 4.40$ & $25.22 \pm 8.49$ & $22.98 \pm 9.54$ & 0.040 & $0.016^{\mathrm{a}}$ \\
\hline
\end{tabular}

Notes: Bold font means significant results. ${ }^{a}$ Indicates a comparison between $\mathrm{HC}$ and meds-off group; ${ }^{\mathrm{b}}$ indicates a comparison between $\mathrm{HC}$ and meds-on group; ${ }^{{ }^{i n d i c a t e s}}$ a comparison between meds-off and meds-on group.

Abbreviations: SL, stride length; GV, gait velocity; CA, cadence; ST, stride time; StPT, stance phase time; SwPT, swing phase time; CV, coefficient of variation; NA, not applicable; ns, no significance.

\section{Correlation Analysis Between Gait Parameters and UPDRS Assessment Scores in the Meds-Off Group}

To explore the influence of different subdomains (bradykinesia, rigidity and rest tremor) on the gait performance of the meds-off group, we conducted Spearman correlation analysis between gait parameters and three subdomain scores (Table 4). Only SL, CV-SL, CV-SwPT, HS, ROM-KJ and ROM-HJ showed differences between $\mathrm{HC}$ and meds-off, thus, we just enrolled these gait parameters into correlation analysis. Bradykinesia scores were significantly correlated with SL ( $r=-0.567 ; p=0.006), \mathrm{CV}-\mathrm{SL}(r=0.544 ; p=0.009)$, ROM-KJ $(r=-0.523 ; p=0.012)$ and ROM-HJ $(r=-0.508 ; p=0.016)$. Moreover, we found significant correlations between rigidity scores and SL $(r=-0.482 ; p=0.023)$, CV-SL $(r=0.570$; $p=0.006)$, and ROM-HJ ( $r=-0.609 ; \mathrm{p}=0.003)$. However, no significant correlation was found between rest tremor scores and gait parameters.

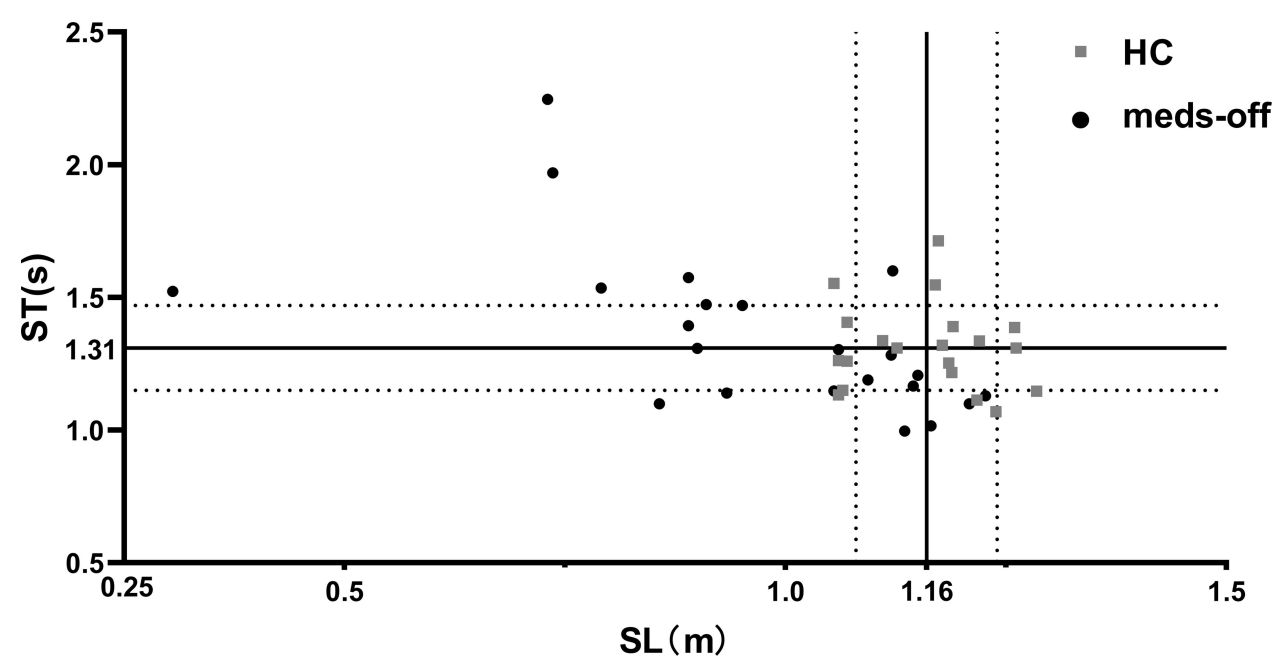

Figure I Walking efficiency analysis of stride length (SL) and stride time (ST). The vertical and horizontal solid line represents the average SL and ST of the HC group, respectively. The two vertical dotted lines represent the average step length minus and plus one standard deviation of the HC group, respectively. The two horizontal dotted lines represent the mean stride time minus and plus one standard deviation of the HC group, respectively. 

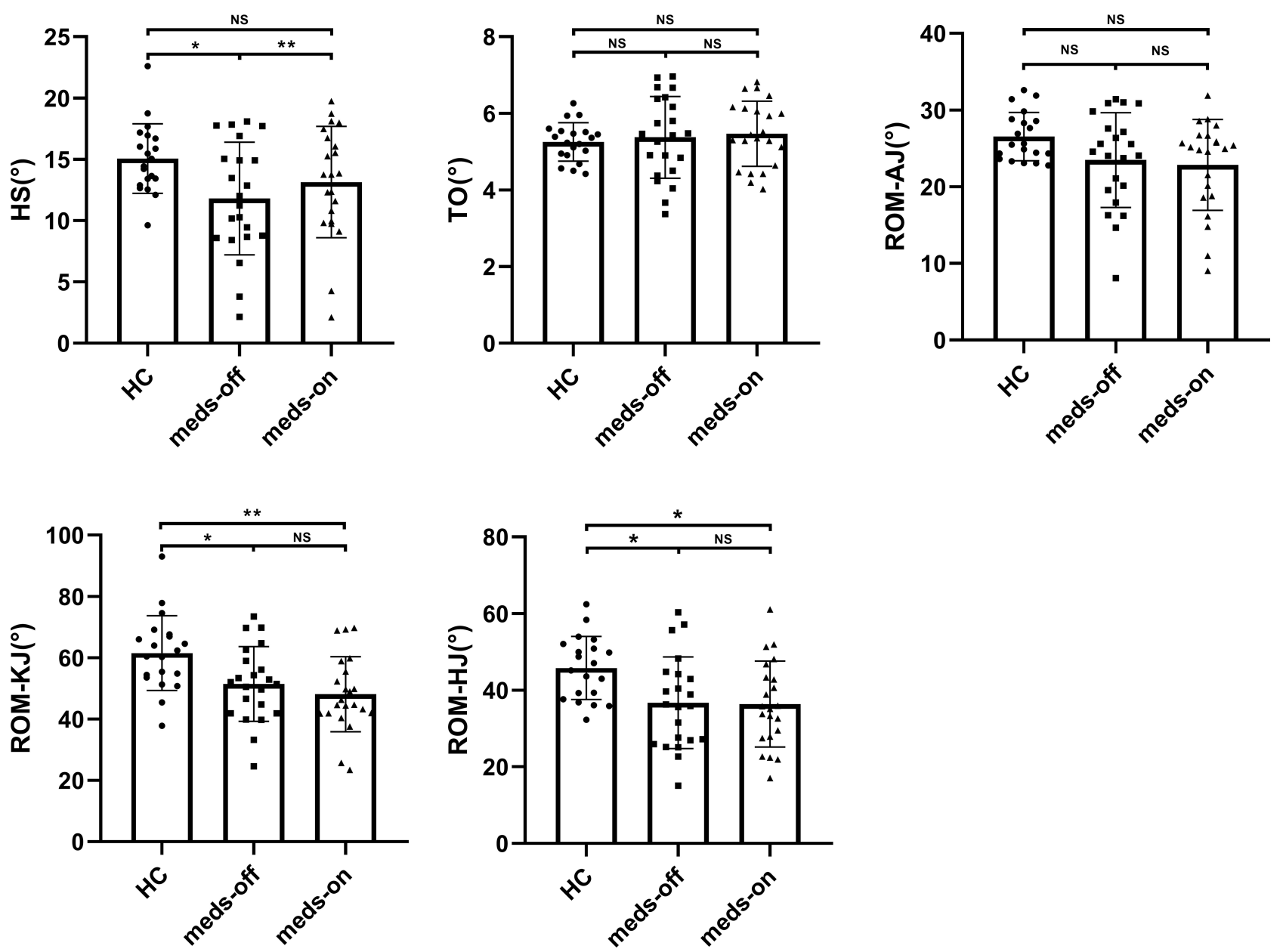

Figure 2 Differences in kinematic gait parameters. * = significant $(p<0.017) ; * *$ highly significant $(p \leq 0.00 I)$.

Abbreviations: HS, heel strike angle; TO, toe-off angle; ROM, range of motion; AJ, ankle joint; KJ, knee joint; HJ, hip joint; NS, no significance.

\section{Discussion}

\section{Gait Analysis Between HC and Meds-Off} Subgroups

The gait performance of MPS have yet to be investigated in depth. Accordingly, the first objective of this research is to comprehensively quantify gait impairments in MPS from the perspective of kinematic and spatiotemporal gait parameters, and the symmetry and variability analyses of gait parameters. To our best knowledge, this study is currently the most comprehensive analysis of the gait performance of old people with MPS.

For spatiotemporal gait parameters, patients with earlystage PD exhibit a shorter SL and a more variable gait pattern. $^{21,23}$ Our research extends previous studies wherein the MPS group exhibit a shorter SL and a more variable gait pattern than HC. ST means the time it takes to walk one stride length. Walking a longer distance in a shorter time indicates a higher walking efficiency. Compared with that of the $\mathrm{HC}$

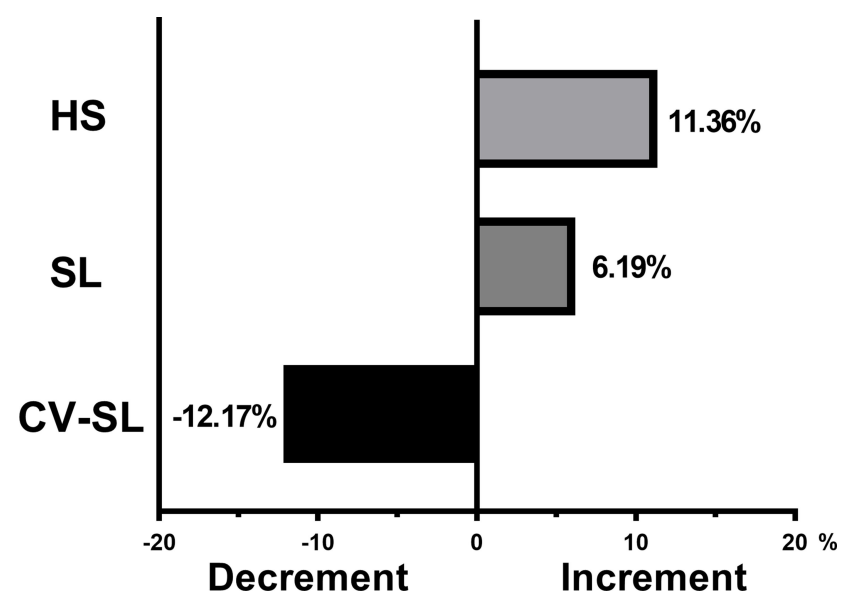

Figure 3 Changes in gait parameters after levodopa/benserazide intake in MPS group.

Abbreviations: HS, heel strike angle; SL, stride length; CV, coefficient of variation.

group, the distribution of SL and ST in the meds-off group is more dispersed and tends to be distributed to the upper left in Figure 1. This result indicates that the walking efficiency of 
Table 3 Symmetry Analysis of Gait Performance

\begin{tabular}{|c|c|c|c|c|c|}
\hline & $\mathrm{HC}$ & Meds-Off & Meds-On & $P$ & Post Hoc Tests \\
\hline Al-SL & $2.24 \pm 0.90$ & $2.67 \pm 2.00$ & $2.87 \pm 2.11$ & 0.603 & NA \\
\hline Al-ST & $6.84 \pm 9.56$ & $10.99 \pm 10.12$ & $9.77 \pm 12.88$ & 0.067 & NA \\
\hline Al-StPT & $|0.99 \pm| 2.9 \mid$ & $14.93 \pm 13.11$ & $14.38 \pm 14.98$ & 0.191 & NA \\
\hline Al-SwPT & $11.65 \pm 20.02$ & $7.75 \pm 6.41$ & $8.23 \pm 7.83$ & 0.984 & NA \\
\hline $\mathrm{Al}-\mathrm{HS}$ & $14.03 \pm 7.72$ & $19.24 \pm 16.77$ & $22.11 \pm 14.87$ & 0.235 & NA \\
\hline Al-TO & $9.85 \pm 6.34$ & $11.40 \pm 11.60$ & $8.19 \pm 9.37$ & 0.503 & NA \\
\hline Al-ROM AJ & $8.61 \pm 7.23$ & $|2.7| \pm 8.45$ & $14.06 \pm 19.53$ & 0.181 & NA \\
\hline Al-ROM KJ & $13.77 \pm 9.92$ & $21.60 \pm 17.88$ & $18.46 \pm 14.10$ & 0.222 & NA \\
\hline Al-ROM HJ & $8.08 \pm 5.59$ & $11.45 \pm 12.54$ & $10.94 \pm 9.04$ & 0.721 & NA \\
\hline
\end{tabular}

Abbreviations: AI, asymmetry index; SL, stride length; ST, stride time; StPT, stance phase time; SwPT, swing phase time; HS, heel strike angle; TO, toe-off angle; ROM, range of motion; AJ, ankle joint; KJ, knee joint; HJ, hip joint; NA, not applicable.

Table 4 Correlation Analysis Between Gait Parameters and UPDRS Assessment Scores

\begin{tabular}{|l|c|c|c|}
\hline & Domain I-Bradykinesia & Domain 2-Rigidity & Domain 3-Rest Tremor \\
\hline SL & $-\mathbf{0 . 5 6 7 ( 0 . 0 0 6 )}$ & $-\mathbf{0 . 4 8 2 ( 0 . 0 2 3 )}$ & $0.195(0.385)$ \\
\hline CV-SL & $\mathbf{0 . 5 4 4 ( 0 . 0 0 9 )}$ & $\mathbf{0 . 5 7 0 ( 0 . 0 0 6 )}$ & $-0.317(0.151)$ \\
\hline CV-SWPT & $0.332(0.132)$ & $0.157(0.484)$ & $-0.292(0.188)$ \\
\hline HS & $-0.359(0.101)$ & $-0.373(0.088)$ & $0.366(0.094)$ \\
\hline ROM-KJ & $-\mathbf{0 . 5 2 3 ( 0 . 0 1 2 )}$ & $-0.333(0.130)$ & $0.114(0.612)$ \\
\hline ROM-HJ & $-\mathbf{0 . 5 0 8 ( 0 . 0 1 6 )}$ & $-\mathbf{0 . 6 0 9 ( 0 . 0 0 3 )}$ & $0.249(0.263)$ \\
\hline
\end{tabular}

Notes: Data were shown as $r(\mathrm{p})$; bold font means significant results.

Abbreviations: SL, stride length; CV, coefficient of variation; SwPT, swing phase time; HS, heel strike angle; ROM, range of motion; KJ, knee joint; HJ, hip joint.

the MPS group decreased. In completing the same walking task, MPS may take more time than HC. The weak push-off of the feet force is responsible for a shorter stride length. ${ }^{24}$ Moreover, short SL and variable gait pattern are associated with imbalance and risk of falling. ${ }^{25,26}$ Although the clinical manifestations of the MPS group are mild, these people are already under the potential risk of falling. For kinematic gait parameters, our research expands previous studies joints movements were decreased in MPS compared with that of HC. The reduction of HS indicates the weakening of the muscle strength of the lower limbs, which may result in falls. ${ }^{27}$ The previous gait analysis of the MPS group did not involve research on the ROM-KJ and ROM-HJ. We found that ROM-KJ and ROM-HJ are decreased in MPS. Similar findings exist in patients with PD. The ROM-KJ of PD patients was significantly lower than that of HC group. And with the progress of PD, the ROM-HJ of PD patients gradually decreases. ${ }^{28} \mathrm{~A}$ smaller extension in the terminal stance result in a decreased ROM-KJ. ${ }^{28}$ Furthermore, an impaired activity of plantar flexors muscles which plays an important role in conducting $\mathrm{KJ}$ extension movement may lead to decreased ROM-KJ. ${ }^{29,30}$ The clinical symptoms of PD are often asymmetric, and this asymmetry has also been confirmed in previous studies. ${ }^{22,31,32}$ In the symmetry analysis of the MPS group, no difference was found in any of those parameters which indicates that MPS group still remains a symmetrical gait pattern. This finding is consistent with the clinical manifestations of MPS because the clinical symptom evaluation of these participants does not exhibit a difference between the left and right sides. To explore the influence of different subdomains (bradykinesia, rigidity and rest tremor) on the gait performance of the meds-off group, 
we conducted Spearman correlation analysis between gait parameters and three subdomain scores. High bradykinesia or rigidity scores were associated with a smaller SL, a more variable gait pattern, and a smaller joint movement. Moreover, no correlation was found between tremor scores and gait parameters.

\section{Changes in Gait Parameters After Levodopa/Benserazide Intake in MPS Group}

It has been well known that the loss of substantia nigra neurons result in PD. However, the etiopathogenesis that underlies MPS remains unknown today. Pigmented neurons of nigra have been reported to decline at a speed of $4.7 \%$ per decade in early adulthood. ${ }^{13}$ To what extent this ageassociated dopamine loss accounts for the development of MPS remains unknown. ${ }^{1}$ If the loss of dopamine leads to the occurrence of MPS, these MPS symptoms should be improved after levodopa/benserazide supplementation. After all, both motor symptoms and gait characteristics of PD can be improved greatly after taking levodopa. ${ }^{26,33}$ Accordingly, the second objective of this research is to explore the effects of levodopa on the gait performance of old individuals with MPS. We conducted ALCT which can be used to predict long-term levodopa responsiveness in the MPS group. ${ }^{16}$ Our findings suggest that we should be conservative about the conclusion that loss of dopamine is the cause of MPS. ${ }^{34-36}$ From a rigorous point of view, the loss of levodopa is at least not the main cause of MPS which is consistent with a recent research. ${ }^{14}$ The reasons are as follow: First, after taking levodopa, the improvement rate of UPDRS part III in the MPS group was only $8.23 \%$ $\pm 6.27 \%$. This value is far below the $30 \%$ improvement rate that supports PD diagnosis. ${ }^{37}$ Second, all the 22 MPS group subjects said no when asked if any improvement was observed after taking levodopa/benserazide. It was reported that levodopa/benserazide worsened balance and postural instability. ${ }^{30}$ However, as the mainstay therapy for PD, levodopa can significantly improve the gait of patients with PD. ${ }^{26,33,38,39}$ Especially for SL and ROM of joints, levodopa/benserazide induced the best improvement and the improvement rate is greater than $50 \% .{ }^{30}$ Contrary to their findings, we found almost no improvement in the gait parameters after ALCT in the MPS group. Both the number of improved gait parameters and the degree of improvement rate are much lower than that of the PD group. In addition, the SL of patients in the MPS group improved after taking the levodopa/benserazide, but it was still lower than that of the HC group. The MPS group slightly benefits from levodopa, suggesting additional mechanisms that underlie MPS. Previous studies have found that MPS is often associated with cognitive decline and dementia. ${ }^{2}$ In addition, a recent research indicated that MPS has a vascular origin and white matter hyperintensities may be responsible for MPS. ${ }^{14}$ On this basis, our research supports the hypothesis that the occurrence of MPS is multifactorial. ${ }^{1,3}$

There are several limitations in this research. First, we had a small sample size, which may limit the power of our estimates. Second, we did not follow up the MPS group, thus, we did not know the final outcome of these patients. In addition, we enrolled UPDRS to determine the presence of MPS. This set of rating scale was originally used to evaluate the motor symptoms of PD. However, no scale has been specifically designed to assess MPS symptoms, and UPDRS is currently the most widely used motor symptom assessment scale.

\section{Conclusion}

Our research expanded previous studies and found that the MPS group exhibited a shorter SL, a more variable but still symmetrical gait pattern and a decreased range of motion of joints compared with the HC group. Although the clinical manifestations of the MPS group are mild, the gait impairment of MPS is already obvious. Contrary to the significant improvement in the gait performance of people with PD, levodopa had minimal effect on the gait performance of old individuals with MPS. Our research will aid the management of elderly individuals with MPS, and shed more light on the pathogenesis of MPS. Subsequent studies can, based on the results of this research, formulate more individualized treatment or rehabilitation programs for MPS group. This will reduce the falling risk and improve the quality of their daily life.

\section{Data Sharing Statement}

The data used to support the findings of this study are available from Pro. Li Zhang.

\section{Statement of Ethics}

Ethical approval was obtained from the Medical Ethics Committee of the Affiliated Brain Hospital of Nanjing Medical University. All participants signed a written informed consent before the experiment. All procedures were in compliance with the declaration of Helsinki. 


\section{Consent for Publication}

There are no conflicts of interest regarding the publication of this manuscript. All authors have read and approved this final version.

\section{Acknowledgments}

This study was funded by 1) Special Funds of the Jiangsu Provincial Key Research and Development Projects [grant NO. BE2018610 and BE2019612], 2) Nanjing Medical Science and technique Development Foundation [grant NO. ZKX17031]. We acknowledge all participants in this research for their understanding and participation. We thank all the researchers of JiBuEn gait analysis system for their timely technical support.

\section{Disclosure}

The authors declare no conflicts of interest regarding this work.

\section{References}

1. Louis ED, Bennett DA. Mild Parkinsonian signs: an overview of an emerging concept. Movement Disorders. 2007;22(12):1681-1688. doi: $10.1002 / \mathrm{mds} .21433$

2. Sasaki S. High prevalence of parkinsonism in patients with MCI or mild Alzheimer's disease. Alzheimer's \& Dementia. 2018;14 (12):1615-1622. doi:10.1016/j.jalz.2018.06.3054

3. Allali G, Verghese J, Mahoney JR. Contributions of mild parkinsonian signs to gait performance in the elderly. Age. 2014;36(4):9678 doi:10.1007/s11357-014-9678-4

4. Prasuhn J, Piskol L, Vollstedt EJ, et al. Non-motor symptoms and quality of life in subjects with mild parkinsonian signs. Acta Neurol Scand. 2017;136(5):495-500. doi:10.1111/ane.12760

5. Fleischman D, Wilson R, Schneider J, Bienias J, Bennett D. Parkinsonian signs and functional disability in old age. Exp Aging Res. 2007;33(1):59-76. doi:10.1080/03610730601006370

6. Lerche S, Brockmann K, Pilotto A, et al. Prospective longitudinal course of cognition in older subjects with mild parkinsonian signs. Alzheimer's Res Ther. 2016;8(1):42. doi:10.1186/s13195-016-0209-7

7. Uemura Y, Wada-Isoe K, Nakashita S, Nakashima KJ. Depression and cognitive impairment in patients with mild parkinsonian signs. Acta Neurologica Scandinavica. 2013;128(3):153-159. doi:10.1111/ ane. 12089

8. Louis E, Tang M, Schupf N, Mayeux R. Functional correlates and prevalence of mild parkinsonian signs in a community population of older people. Arch Neurol. 2005;62(2):297-302. doi:10.1001/ archneur.62.2.297

9. Waite L, Grayson D, Piguet O, Creasey H, Bennett H, Broe G. Gait slowing as a predictor of incident dementia: 6-year longitudinal data from the Sydney Older Persons Study. J Neurol Sci. 2005;229230:89-93. doi:10.1016/j.jns.2004.11.009

10. Mahoney J, Verghese J, Holtzer R, Allali G. The evolution of mild parkinsonian signs in aging. $J$ Neurol. 2014;261(10):1922-1928. doi:10.1007/s00415-014-7442-4

11. Palmisano C, Brandt G, Pozzi NG, et al. Sit-to-walk performance in Parkinson's disease: a comparison between faller and non-faller patients. Clin Biomechanics. 2019;63:140-146. doi:10.1016/j. clinbiomech.2019.03.002
12. Palmisano C, Brandt G, Vissani M, et al. Gait initiation in Parkinson's disease: impact of dopamine depletion and initial stance condition. Front Bioeng Biotechnol. 2020;8:137. doi:10.3389/fbioe.2020.00137

13. Fearnley J, Lees A. Ageing and Parkinson's disease: substantia nigra regional selectivity. Brain. 1991;114(5):2283-2301. doi:10.1093/ brain/114.5.2283

14. Rosso AL, Bohnen NI, Launer LJ, Aizenstein HJ, Yaffe K, Rosano C. Vascular and dopaminergic contributors to mild parkinsonian signs in older adults. Neurology. 2018;90(3):e223-e229. doi:10.1212/ WNL.0000000000004842

15. Seiffert P, Derejczyk J, Kawa J, et al. Frailty phenotype and the role of levodopa challenge test in geriatric inpatients with mild parkinsonian signs. Biogerontology. 2017;18(4):641-650. doi:10.1007/s10522-0179716-6

16. Merello M, Nouzeilles MI, Arce GP, Leiguarda R. Accuracy of acute levodopa challenge for clinical prediction of sustained long-term levodopa response as a major criterion for idiopathic Parkinson's disease diagnosis. Movement Disorders. 2002;17(4):795-798. doi: $10.1002 / \mathrm{mds} .10123$

17. Tao S, Zhang X, Cai H, Lv Z, Hu C, Xie H. Gait based biometric personal authentication by using MEMS inertial sensors. J Ambient Intell Humaniz Comput. 2018;9(5):1705-1712. doi:10.1007/s12652-018-0880-6

18. Mancini M, King L, Salarian A, Holmstrom L, McNames J, Horak FB. Mobility lab to assess balance and gait with synchronized body-worn sensors. J Bioeng Biomed Sci. 2011;Suppl 1:007.

19. Galna B, Lord S, Rochester L. Is gait variability reliable in older adults and Parkinson's disease? Towards an optimal testing protocol. Gait Posture. 2013;37(4):580-585. doi:10.1016/j.gaitpost.2012.09.025

20. Patterson KK, Gage WH, Brooks D, Black SE, Mcllroy WE. Evaluation of gait symmetry after stroke: a comparison of current methods and recommendations for standardization. Gait Posture. 2010;31(2):241-246. doi:10.1016/j.gaitpost.2009.10.014

21. Zhang M, Artan NS, Gu H, et al. Gait study of Parkinson's disease subjects using haptic cues with a motorized walker. Sensors. 2018;18:10.

22. Serrao M, Chini G, Caramanico G, et al. Prediction of responsiveness of gait variables to rehabilitation training in Parkinson's disease. Front Neurol. 2019;10:826. doi:10.3389/fneur.2019.00826

23. Hobert MA, Nussbaum S, Heger T, Berg D, Maetzler W, Heinzel S. Progressive gait deficits in Parkinson's disease: a wearable-based biannual 5-year prospective study. Front Aging Neurosci. 2019;11:22. doi:10.3389/fnagi.2019.00022

24. Burleigh-Jacobs A, Horak FB, Nutt JG, Obeso JA. Step initiation in Parkinson's disease: influence of levodopa and external sensory triggers. Movement Disorders. 1997;12(2):206-215. doi:10.1002/ mds. 870120211

25. Hausdorff JM. Gait dynamics, fractals and falls: finding meaning in the stride-to-stride fluctuations of human walking. Hum Mov Sci. 2007;26(4):555-589. doi:10.1016/j.humov.2007.05.003

26. Smulders K, Dale ML, Carlson-Kuhta P, Nutt JG, Horak FB. Pharmacological treatment in Parkinson's disease: effects on gait. Parkinsonism Relat Disord. 2016;31:3-13. doi:10.1016/j. parkreldis.2016.07.006

27. Schlachetzki JCM, Barth J, Marxreiter F, et al. Wearable sensors objectively measure gait parameters in Parkinson's disease. PLoS One. 2017;12(10):e0183989. doi:10.1371/journal.pone.0183989

28. Dipaola M, Pavan EE, Cattaneo A, et al. Mechanical energy recovery during walking in patients with Parkinson disease. PLoS One. 2016;11(6):e0156420. doi:10.1371/journal.pone.0156420

29. Kimmeskamp S, Hennig EM. Heel to toe motion characteristics in Parkinson patients during free walking. Clin Biomechanics. 2001;16 (9):806-812. doi:10.1016/S0268-0033(01)00069-9

30. Mitoma H, Hayashi R, Yanagisawa N, Tsukagoshi H. Characteristics of parkinsonian and ataxic gaits: a study using surface electromyograms, angular displacements and floor reaction forces. J Neurol Sci. 2000;174(1):22-39. doi:10.1016/S0022-510X(99)00329-9 
31. Koh SB, Park YM, Kim MJ, Kim WS. Influences of elbow, shoulder, trunk motion and temporospatial parameters on arm swing asymmetry of Parkinson's disease during walking. Hum Mov Sci. 2019;68:102527. doi:10.1016/j.humov.2019.102527

32. Rehman RZU, Del Din S, Guan Y, Yarnall AJ, Shi JQ, Rochester L. Selecting clinically relevant gait characteristics for classification of early Parkinson's disease: a comprehensive machine learning approach. Sci Rep. 2019;9(1):17269. doi:10.1038/s41598-01953656-7

33. Curtze C, Nutt JG, Carlson-Kuhta P, Mancini M, Horak FB. Levodopa is a double-edged sword for balance and gait in people with Parkinson's disease. Movement Disorders. 2015;30 (10):1361-1370. doi:10.1002/mds.26269

34. Louis E, Tang M, Mayeux R. Factor structure of parkinsonian signs in the community-dwelling elderly. Movement Disorders. 2004;19 (3):268-272. doi:10.1002/mds.20013

35. Buchman A, Shulman J, Nag S, et al. Nigral pathology and parkinsonian signs in elders without Parkinson disease. Ann Neurol. 2012;71(2):258-266. doi:10.1002/ana.22588
36. Horvath J, Burkhard P, Herrmann F, Bouras C, Kövari E. Neuropathology of parkinsonism in patients with pure Alzheimer's disease. J Alzheimer's Dis. 2014;39(1):115-120. doi:10.3233/JAD131289

37. Postuma RB, Berg D, Stern M, et al. MDS clinical diagnostic criteria for Parkinson's disease. Movement Disorders. 2015;30 (12):1591-1601. doi:10.1002/mds.26424

38. Foreman KB, Singer ML, Addison O, Marcus RL, LaStayo PC, Dibble LE. Effects of dopamine replacement therapy on lower extremity kinetics and kinematics during a rapid force production task in persons with Parkinson disease. Gait Posture. 2014;39(1):638-640. doi:10.1016/j.gaitpost.2013.07.114

39. Fabbri M, Coelho M, Abreu D, et al. Do patients with late-stage Parkinson's disease still respond to levodopa? Parkinsonism Relat Disord. 2016;26:10-16. doi:10.1016/j.parkreldis.2016.02.021
Risk Management and Healthcare Policy

\section{Publish your work in this journal}

Risk Management and Healthcare Policy is an international, peerreviewed, open access journal focusing on all aspects of public health, policy, and preventative measures to promote good health and improve morbidity and mortality in the population. The journal welcomes submitted papers covering original research, basic science, clinical \& epidemiological studies, reviews and evaluations,
Dovepress

guidelines, expert opinion and commentary, case reports and extended reports. The manuscript management system is completely online and includes a very quick and fair peer-review system, which is all easy to use. Visit http://www.dovepress.com/testimonials.php to read real quotes from published authors. 